\title{
Ultrastructural localization of the Pasteurella multocida toxin in a toxin- producing strain
}

\author{
Christine iDali, ${ }^{1}$ Niels T. Foged,${ }^{2}$ Peer L. Frandsen, ${ }^{2}$ Morten H. Nielsen ${ }^{1}$ and \\ FOLMER ELLING ${ }^{1}$ \\ ${ }^{1}$ Institute of Pathological Anatomy, University of Copenhagen 11, Frederik d. V's Vej, DK-2100 Copenhagen, Denmark \\ ${ }^{2}$ Animal Biotechnology Research Centre, National Veterinary Laboratory, Copenhagen, Denmark
}

(Received 21 November 1990; revised 14 January 1991; accepted 21 January 1990)

\begin{abstract}
Toxigenic strains of Pasteurella multocida produce the $147 \mathrm{kDa}$ protein Pasteurella multocida toxin (PMT) which is responsible for the osteoclastic bone resorption in progressive atrophic rhinitis in pigs and induces such resorption in all experimental animals tested so far. In the present study we have carried out immunocytochemistry on formaldehyde- and glutaraldehyde-fixed ultracryocut $P$. multocida using a pool of monoclonal antibodies against different epitopes on PMT as the first layer and affinity purified rabbit anti-mouse IgG as the second layer. Goat anti-rabbit IgG conjugated with $5 \mathrm{~nm}$ gold particles was used as marker. The gold particles were silverenhanced prior to examination in the transmission electron microscope. Whole bacteria were also immunostained after fixation and critical point drying and examined by scanning transmission electron microscopy. The results showed that PMT was located in the cytoplasm of $P$. multocida. PMT could not be detected on intact, undamaged $P$. multocida by scanning electron microscopy. Neither pili nor flagella could be detected on the surface of the negatively stained $\boldsymbol{P}$. multocida strains investigated. PMT has a series of characteristics encompassed in the definition of an exotoxin. However, that PMT was not secreted by living intact $P$. multocida is unexpected for an exotoxin.
\end{abstract}

\section{Introduction}

Inoculation of toxigenic strains of Pasteurella multocida in the nasal cavity of young piglets pretreated with a mild acetic acid solution allows the bacteria to establish themselves, grow in the nasal cavity and induce turbinate atrophy (Pedersen \& Elling, 1984; Elling \& Pedersen, 1985). Toxigenic strains of $P$. multocida produce a protein, Pasteurella multocida toxin (PMT) which has been purified and characterized (Nakai \& Kume, 1984; Foged et al., 1987). Intranasal instillation of PMT will induce resorption of nasal bone structures after $24 \mathrm{~h}$ in newborn rats. The induced lesions were similar to spontaneous progressive atrophic rhinitis in pigs, indicating that PMT is the bacterial product responsible for bone resorption in this disease (Foged et al., 1987). It has been debated whether toxigenic $P$. multocida possess pili facilitating adherence to the nasal mucosa at infection (Trigo \& Pijoan, 1988; Nakai et al., 1988). The aim of the present study was to visualize the localization of PMT, both on the surface of toxigenic P. multocida and in the cells, by the use of immunocytochemistry as an ultra-

Abbreviation: PMT, Pasteurella multocida toxin. structural approach to the elucidation of PMT production. It was also intended to determine whether toxigenic strains of $P$. multocida subsp. multocida, capable of inducing progressive atrophic rhinitis in pigs, possess pili.

\section{Methods}

A toxin-producing strain of $P$. multocida subsp. multocida (NCTC 12178) was grown overnight on blood agar plates. The bacteria were harvested from the agar plates and suspended in $0.01 \mathrm{M}$-PBS $\left(8 \mathrm{~g} \mathrm{I}^{-1}\right.$ $\mathrm{NaCl}, 0.2 \mathrm{~g} \mathrm{l}^{-1} \mathrm{KCl}, 1.44 \mathrm{~g}^{-1} \quad \mathrm{Na}_{2} \mathrm{HPO}_{4} .2 \mathrm{H}_{2} \mathrm{O}, 0.23 \mathrm{~g}^{-1} \mathrm{NaH}_{2}$ $\mathrm{PO}_{4} \cdot \mathrm{H}_{2} \mathrm{O}, \mathrm{pH} 7 \cdot 2$ ) and centrifuged at $10000 \mathrm{~g}$ for $10 \mathrm{~min}$. They were then fixed in $0.5 \%$ formaldehyde and $0.5 \%$ glutaraldehyde in $0.1 \mathrm{M}$ phosphate buffer ( $\mathrm{pH} \mathrm{7.2)} \mathrm{for} 15 \mathrm{~min}$ and subsequently centrifuged at $10000 \mathrm{~g}$ for $10 \mathrm{~min}$. The pellet was washed in PBS for $4 \times 5 \mathrm{~min}$ and stabilized in $10 \%(\mathrm{w} / \mathrm{v})$ phosphate buffered gelatine for $20 \mathrm{~min}$ followed by centrifugation at $10000 \mathrm{~g}$ for $15 \mathrm{~min}$, all at $37^{\circ} \mathrm{C}$. Droplets of the pellet were placed on an ice-cooled object glass and hardened for $2 \mathrm{~min}$, then covered by another object glass and hardened on ice for $30 \mathrm{~min}$. The upper glass was removed and each droplet was cut into slices, cryoprotected in $2.3 \mathrm{M}$-sucrose and $1 \%(\mathrm{w} / \mathrm{v})$ formaldehyde PBS solution for $20 \mathrm{~min}$, divided into blocks $(1 \times 1 \times 1 \mathrm{~mm})$ that were mounted on silver stubs with Tissuetec (Miles Inc.) and frozen in liquid nitrogen. Sections of $100 \mathrm{~nm}$ were made with a RMC MT6000 X-L cryoultramicrotome at $-80^{\circ} \mathrm{C}$. Sections were transferred to a droplet 
of $1.8 \mathrm{M}$-sucrose in PBS, and then to Formvar-coated, carbonized 200 mesh nickel grids. The grids were floated on droplets of PBS with $0.2 \%$ bovine serum albumin (BSA) and $1 \%(\mathrm{v} / \mathrm{v})$ goat serum, and kept overnight in $1 \%(\mathrm{w} / \mathrm{v})$ formaldehyde in PBS at $4{ }^{\circ} \mathrm{C}$. They were then washed in PBS for 20 min, placed on a droplet of $0.02 \mathrm{M}$-glycine in PBS for $10 \mathrm{~min}$, transferred to droplets of PBS containing $0.2 \%$ BSA ( $\mathrm{pH} \mathrm{7.2)} \mathrm{and} \mathrm{washed} 4 \times 5 \mathrm{~min}$, and finally floated on $10 \%(\mathrm{v} / \mathrm{v})$ goat serum in PBS containing $0.2 \%$ BSA (pH 7.2) for 20 min. The sections were incubated for $30 \mathrm{~min}$ with the primary antibody, i.e. a pool of 10 monoclonal antibodies against different epitopes on PMT (Foged, 1988). The pool contained $100 \mu \mathrm{g} \mathrm{ml}^{-1}$ of each antibody and it was diluted $1: 1000$ in PBS containing $0.25 \%$ BSA (pH 7.2). The grids were washed $4 \times 5 \mathrm{~min}$ in PBS, then incubated with affinity-purified rabbit anti-mouse IgG (Dakopatts, Denmark, $10 \mu \mathrm{g} \mathrm{ml}^{-1}$ ) diluted 1:30 in PBS containing $1 \%(\mathrm{v} / \mathrm{v})$ goat serum and $0.2 \%$ BSA $(\mathrm{pH} \mathrm{7.2)}$ for $20 \mathrm{~min}$ and washed $4 \times 5 \mathrm{~min}$ as described above. The grids were subsequently washed for $5 \mathrm{~min}$ in $0.05 \mathrm{M}$-Tris-buffered-saline, pH 8.2 (TBS), and transferred to droplets containing goat anti-rabbit IgG conjugated with $5 \mathrm{~nm}$ gold particles (Janssen Pharma) diluted 1:50 in 0.02 M-TBS and incubated for $20 \mathrm{~min}$. The grids were washed $4 \times 5 \mathrm{~min}$ in $0.05 \mathrm{M}$-TBS and for $5 \mathrm{~min}$ in $0.1 \mathrm{M}$-phosphate buffer $\mathrm{pH} 7 \cdot 4$. The sections were fixed on droplets with $2 \%(\mathrm{v} / \mathrm{v})$ glutaraldehyde in $0 \cdot 1 \mathrm{M}$-phosphate buffer $\mathrm{pH} 7.4$ for $10 \mathrm{~min}$, washed in distilled water $(4 \times 5 \mathrm{~min})$ and then silver-enhanced for $7 \mathrm{~min}$ in a physical developer containing $0.11 \%$ silver lactate, $0.85 \%$ hydroquinone and $15 \%(\mathrm{w} / \mathrm{v})$ gum arabic in citrate buffer pH 3.5 (Bastholm et al., 1986). The sections were contrasted either (1) by negative staining with $0.5 \%$ phosphotungstic acid (PTA) in distilled water on ice in a humid chamber for $5 \mathrm{~min}$; or (2) with ice cold droplets of $2 \%(\mathrm{w} / \mathrm{v})$ methylcellulose in distilled water with $0.4 \%$ uranyl acetate for $5 \mathrm{~min}$; or (3) with $3 \%(\mathrm{w} / \mathrm{v})$ polyvinyl alcohol in distilled water containing $0.3 \%$ uranyl acetate (Tokuyasu, 1986). Excess stain was removed with filter paper, and the grids were airdried.

A nontoxigenic type D strain of $P$. multocida subsp. multocida (ATTC 7707) was used as a control. In order to reveal possible nonspecific binding of the secondary and tertiary antibody to bacterial components, the first layer was omitted. As a further control, staining was done with primary monoclonal antibody absorbed with PMT.

In order to evaluate the PMT concentration and the morphology of $P$. multocida and the presence of PMT during a $30 \mathrm{~h}$ observation period, a fresh culture of $P$. multocida was prepared from the toxin-producing strain NCTC 12178. A loop of bacteria was harvested from an agar plate and inoculated in $80 \mathrm{ml}$ of Trypticase Soy Broth (BBL). After $3 \mathrm{~h}$, $1 \mathrm{ml}$ of culture was transferred to $200 \mathrm{ml}$ broth and kept at $37^{\circ} \mathrm{C}$. The number of bacteria was measured as the $\mathrm{OD}_{450}$ of $1 \mathrm{ml}$ of the bacterial culture inoculation in broth, 10,20 and $30 \mathrm{~h}$ after initiation of the experiment.

The remainder of the bacterial culture was centrifuged at $10000 \mathrm{~g}$ for $30 \mathrm{~min}$. The PMT concentration of the supernatant was determined by ELISA (Foged, 1988). Part of the pellet was suspended in $4 \mathrm{ml} \mathrm{PBS}$ and after sonication, the amount of toxin was quantified as described above; another part of the pellet was prepared for cryosection as described above.

For transmission electron microscopy (TEM) and scanning electron microscopy (SEM), whole bacteria were immunostained as above, then fixed in glutaraldehyde and silver-enhanced for $40 \mathrm{~min}$ as above (Scopsi et al., 1986). For TEM the grids were washed with distilled water and air-dried. For SEM they were ethanol-dehydrated, critical point dried, and sputtercoated with a $2 \mathrm{~nm}$ layer of gold/palladium. TEM, SEM and scanning transmission electron microscopy (STEM) was performed in a JEOL $100 \mathrm{C}$ electron microscope equipped with EM-ASID.

For ultrastructural examination of the possible presence of pili and flagella on the surface whole, formaldehyde fixed bacteria were negatively stained with PTA and examined in TEM.
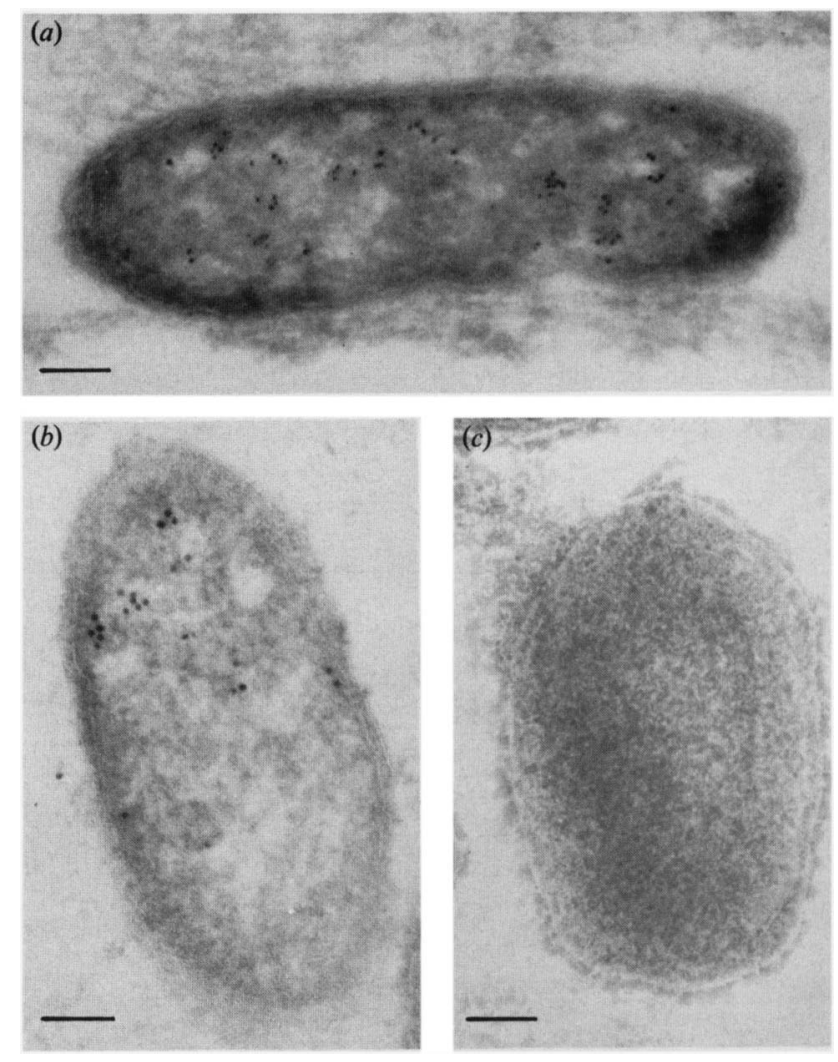

Fig. 1. Electron micrographs of ultracryocut $P$. multocida. (a) A representative toxigenic strain of $P$. multocida subsp. multocida (NCTC 12178) stained with a pool of monoclonal antibodies against the PMT as the first layer, rabbit anti-mouse IgG as the second, and goat antirabbit IgG conjugated with $5 \mathrm{~nm}$ colloidal gold particles as the third layer. (b) As (a), but with a lower density of gold particles. (c) Representative nontoxigenic strain of $P$. multocida subsp. multocida stained as above. The gold particles were silver-enhanced. Specimens were positively stained with methylcellulose containing uranyl acetate. PMT appears to be distributed almost exclusively within the inner membrane of the bacterial wall. Bar, $200 \mathrm{~nm}$.

\section{Results}

The localization of PMT was demonstrated on aldehydefixed cryosectioned $P$. multocid $a$ by a three-layer indirect immuno-cytochemical technique employing a pool of monoclonal antibodies as the first layer and $5 \mathrm{~nm}$ goldconjugated IgG as third layer. Owing to the overall high electron density of the ultracryocut bacteria, silverenhancement of the gold labels greatly facilitated the detection of immunolabelling. Labelling for PMT was almost exclusively found over the bacterial cytoplasm, as only $2.3 \%$ of the gold particles were noted over the outer membranes (Fig. 1a). Gold particles were evenly distributed within the cytoplasm. The labelling density varied from bacterium to bacterium, ranging from 3 to 70 gold particles (Fig. 1a,b). Approximately 8000 bacteria were examined. 


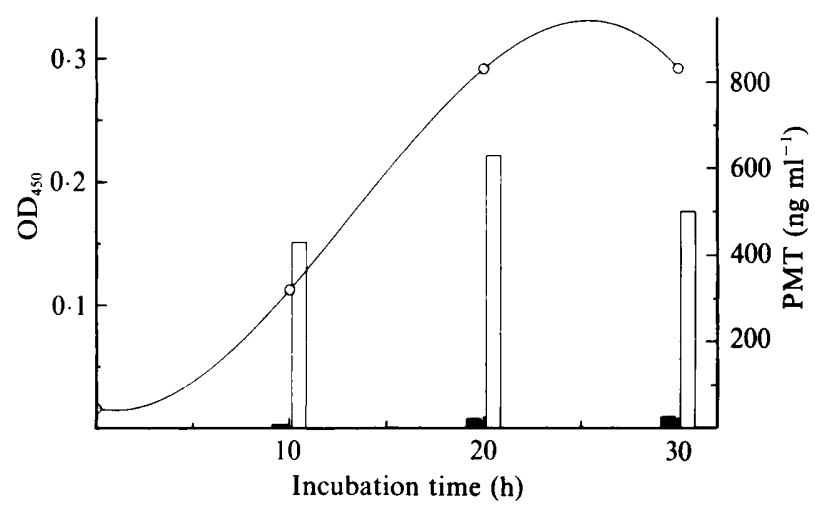

Fig. 2. Production of PMT at different times during the growth of a non-shaken culture of toxigenic $P$. multocida ssp. multocida. (O) Bacterial growth estimated as the background-corrected $\mathrm{OD}_{450}$. Solid bars indicate PMT concentration in the $200 \mathrm{ml}$ of culture supernatant; open bars show PMT concentration in the $4 \mathrm{ml}$ of resuspended sonicated cell pellet.

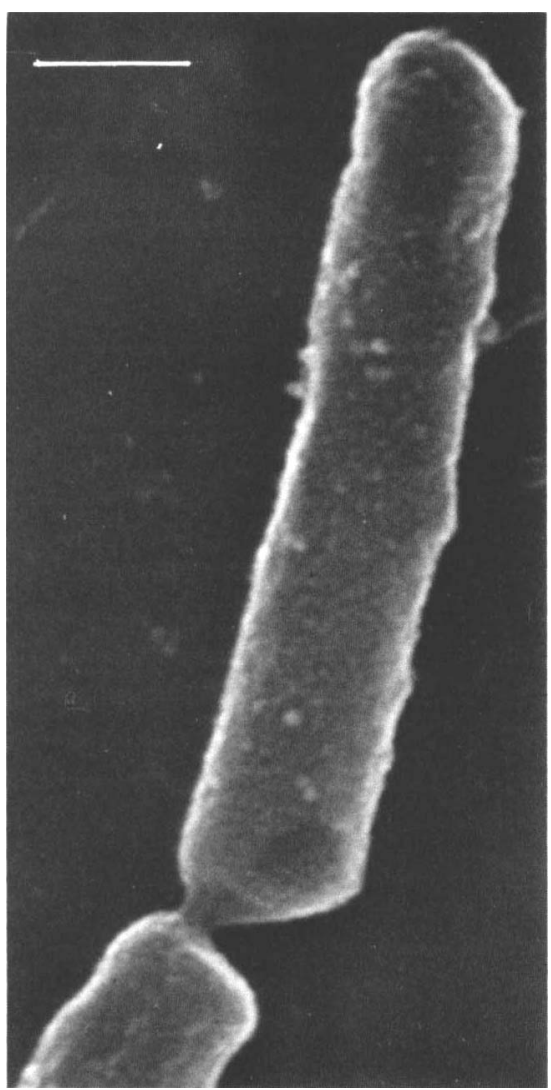

Fig. 3. Scanning electron micrograph of a representative whole toxigenic strain of $P$. multocida ssp. multocida fixed and immunostained as above but silver-enhanced for a longer period of time. Apart from occasional granular material, the bacterial surface is smooth. Bar, $0.5 \mu \mathrm{m}$.

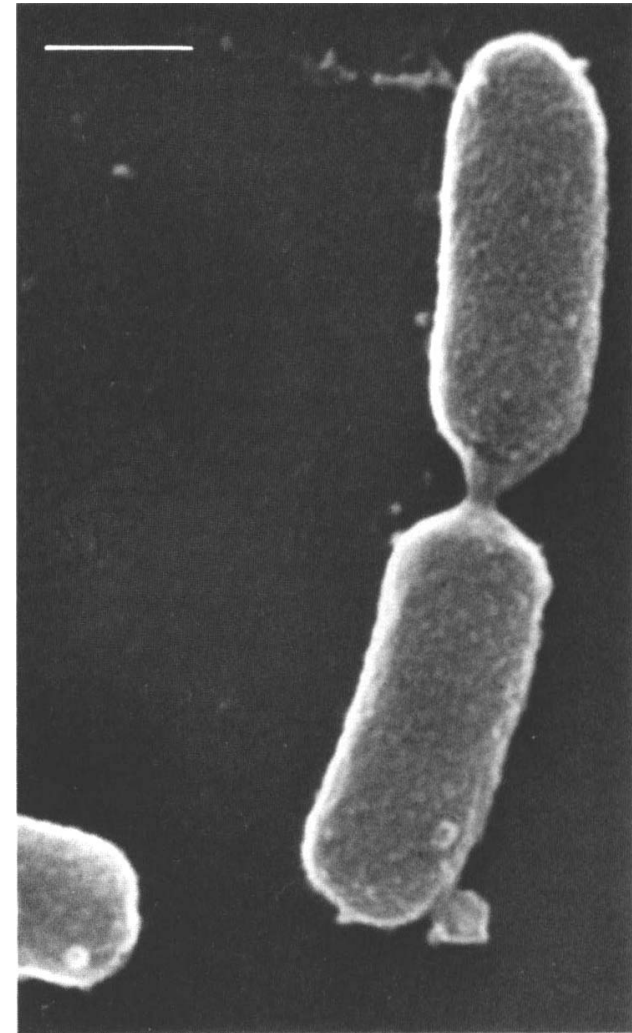

Fig. 4. Scanning electron micrograph of a representative whole nontoxigenic $P$. multocida ssp. multocida. Apart from occasional granular material, the bacterial surface is smooth. Bar, $0.5 \mu \mathrm{m}$.

There was no labelling for PMT on the nontoxigenic $P$. multocida strain (Fig. 1c) and the PMT-absorbed monoclonal antibodies showed no labelling of the toxigenic strain. Additionally, negative labelling was obtained by omitting the first layer.

There was no obvious difference in immune labelling density for PMT on the ultracryosectioned bacteria or changes of ultrastructural morphology at different times during the $30 \mathrm{~h}$ observation period. Furthermore, the PMT concentration in the pellet of bacteria as determined by ELISA increased only slightly during the same period (Fig. 2), as did the very low concentration in the supernatant.

TEM and SEM examination of whole P. multocida, untreated or treated with detergent did not reveal PMT on the surface of intact undamaged $P$. multocida (Figs 3 , 4). TEM and SEM examination confirmed the light microscopical observations that the shape of the nontoxigenic $P$. multocida strain appeared more rounded or coccoid than the toxigenic strain (Figs $1 a, b, c, 3,4)$.

Pili or flagella were not observed on the surface of the negatively stained $P$. multocida examined by TEM (Fig. $5)$. 


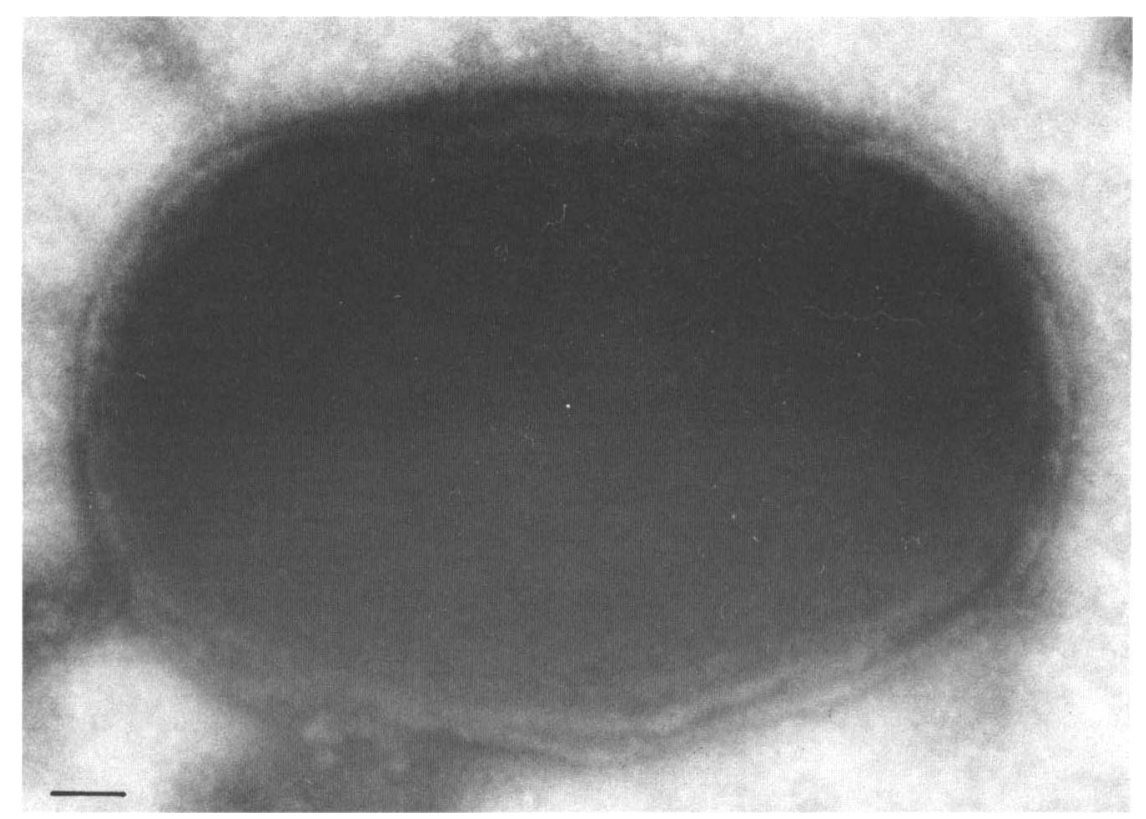

Fig. 5. Transmission electron micrograph of a representative negatively stained toxigenic $P$. multocida. Neither flagella nor pili could be detected on the surface of the bacteria. Bar, $100 \mathrm{~nm}$.

\section{Discussion}

We have demonstrated that the outer membranes of $P$. multocida can be easily distinguished by ultrathin cryosectioning, whereas the intracellular details of substructure, e.g. ribosomes, are less distinct than normally observed in ultrathin-sectioned resin-embedded bacteria. We found that positive contrasting with methylcellulose-uranyl or PVA-uranyl was superior to negative staining with PTA when visualizing membranes and cytoplasm of $P$. multocida. Cryoprotection with sucrose prior to freezing in liquid nitrogen eliminated problems with breakdown of the bacterial membranes previously encountered by investigators preparing $P$. multocida for electron microscopy using plastic embedding (Günther et al., 1986).

Previous investigations have shown that strains of $P$. multocida associated with atrophic rhinitis possessed pili on the surface (Trijo \& Pijoan, 1988). The toxigenic strain and the nontoxigenic strain in our experiment did not show pili or flagella on the surface of negatively stained whole bacteria by TEM.

The present study appears to be the first visualizing the fine structural localization of PMT in a toxigenic strain of $P$. multocida. The chemical and biological properties of PMT indicate that it is an exotoxin. There are no indications, however, of PMT being excreted by living bacteria, which is considered a major feature in the differentiation between exo- and endotoxins (Jawetz et al., 1989). Thus, PMT was identified only in the bacterial cytoplasm and was rarely localized adjacent to the outer membrane on the ultracryocut $P$. multocida. Similarly, it was not identified on the surface of immunostained whole bacteria examined by TEM or SEM. The results clearly indicated that PMT is a cytoplasmic constituent and not a structural component of the bacterial cell wall as are endotoxins (Braude et al., 1986). The results of the TEM and SEM examination of immunostained whole bacteria are significant and corroborate previous observations where PMT could only be localized on unfixed bacteria that had been partially lysed by washing in distilled water prior to staining on grids, and not on intact bacteria (L. Bastholm \& F. Elling, unpublished results). The immunocytochemical findings and the determination of PMT concentration at different times during the $30 \mathrm{~h}$ observation period are consistent with the observation that PMT could only be detected in appreciable amounts in the supernatant of a broth-grown $P$. multocida culture after disintegration of the bacteria by sonication (Nakai et al., 1985), and also indicate that PMT will not be released from intact $P$. multocida. Consequently, it can be argued that excretion from living bacteria is not an essential characteristic for all 'exotoxins'.

Christine iDali is a 'Lundbeck Scholar'. The authors thank Lone Bastholm and Søren Munk Jensen for valuable assistance. This study was supported by the Danish Medical Research Council, grant no. 127125, the Danish Veterinary and Agricultural Research Council, grant 
no. 13-4207, 'Erik Hørslev og hustru Birgit Hørslevs Fond' and 'Grethe og Hans Lundbeck's Legat'.

\section{References}

BASTholm, L., SCOPSI, L. \& Nielsen, M. H. (1986). Silver-enhanced immunogold staining of semithin and ultrathin cryosections. Journal of Electron Microscopy Technique 4, 175-176.

Braude, A. I., Davis, C. E. \& Fierer, J. (1986). Infectious Diseases and Medical Microbiology, pp. 42-59. Philadelphia: W. B. Saunders Company.

Elling, F. \& Pedersen, K. B. (1985). The pathogenesis of persistent turbinate atrophy induced by toxigenic Pasteurella multocida in pigs. Veterinary Patholology 22, 469-474.

Foged, N. T., Pedersen, K. B. \& Elling, F. (1987). Characterization and biological effects of the Pasteurella multocida toxin. FEMS Microbiology Letters 43, 45-51.

FOGED, N. T. (1988). Quantitation and purification of the Pasteurella multocida toxin by using monoclonal antibodies. Infection and Immunity 56, 1901-1906.

GÜNTHER, H., ROSNER, H., GodaT, M. \& ERLER, W. (1986). Electron microscopic visualization of the capsule of Pasteurella. Acta histochemica, Suppl. Band. XXXIII, 293-296.
JAWEtZ, E., Melnick, J. L., Adelberg, E. A., Brooks, G. F., Butel, J. S. \& ORnston, L. N. (1989). Medical Microbiology, pp. 128-129. New York: Prentice-Hall.

Nakai, T., Sawata, A., Tsuil, M. \& Kume, K. (1984). Purification of dermonecrotic toxin from a sonic extract of Pasteurella multocida SP-72 serotype D. Infection and Immunity 46, 429-434.

Nakai, T., Kume, K., Yoskikawa, H., Oyamada, T. \& Yoshikawa, T. (1988). Adherence of Pasteurella multocida or Bordetella bronchiseptica to the swine nasal epithelial cell in vitro. Infection and Immunity 56, 234-240.

NaKaI, T., Sawata, A. \& Kume, K. (1985). Intracellular locations of dermonecrotic toxins in Pasteurella multocida and in Bordetella bronchiseptica. American Journal of Veterinary Research 46, no. 4.

Pedersen, K. B. \& Elling, F. (1984). The pathogenesis of atrophic rhinitis in pigs induced by toxigenic Pasteurella multocida. Journal of Comparative Pathology 94, 203-214.

Scopsi, L., Larsson, L.-I., Bastholm, L. \& Nielsen, M. N. (1986). Silver-enhanced colloidal gold probes as markers for scanning electron microscopy. Histochemistry 86, 35-41.

TokUYASU, K. T. (1986). Application of cryoultramicrotomy to immunocytochemistry. Journal of Microscopy 143, 139-149.

Trigo, E. \& Pijoan, C. (1988). Presence of pili in Pasteurella multocida strains associated with atrophic rhinitis. Veterinary Record 122, 19. 